\title{
Ameliorative Potential of Allium cepa Lam. Leaves on Diabetes Induced and Chronic Constriction Injury Induced Neuropathic Pain in Experimental Rats
}

\author{
Dureshahwar Khan ${ }^{1}$, Mubashir Mohammed ${ }^{1}$, Aman Upaganlawar², Chandrashekhar D. Upasani², \\ Hemant D. Une ${ }^{1, *}$ \\ 1'Department of Pharmacology, Y. B. Chavan College of Pharmacy, Dr. Rafiq Zakaria Campus, Dr. Rafiq Zakaria Marg, Rauza Bagh, \\ Aurangabad, Maharashtra, INDIA. \\ 2Department of Pharmacology, SNJB's SSDJ College of Pharmacy, Neminagar, Chandwad, Maharashtra, INDIA.
}

\begin{abstract}
Background: Neuropathy can be induced in rats by peripheral injuries, depending on compression of complete or a section of sciatic nerve and chemically by Streptozotocin (STZ). Materials and Methods: In the present study neuropathic pain were induced in rats by two methods, chronic constriction injury (surgical model) and STZ $(40 \mathrm{mg} / \mathrm{kg} / \mathrm{i} . \mathrm{p}$.) induced diabetes (drug induced model). In both the models, behavioural as well as markers of oxidative stress were studied. Behavioural parameters were tested using vonfrey hair and Randall Selitto analgesiometer whereas biochemical parameter includes glycosylated haemoglobin and markers of oxidative stress. The study was further supported by histopathology of sciatic nerve. Flavonoid rich extract of Allium cepa Lam. leaves was administered at three different doses viz. 25, 50 and $100 \mathrm{mg} / \mathrm{kg} / \mathrm{p} .0$ to the rats with neuropathic pain. Both the models of neuropathic pain showed significant alteration in behavioural as well as oxidative stress parameters. Results: Treatment of Allium cepa leaves extract showed dose dependent improvement in behavioural and biochemical parameters towards normal ( $p$ value $<0.001,<0.05$ and $<0.01$ ). The altered histopathological changes in sciatic nerve were also significantly improved as compared to rats with neuropathic pain. Conclusion: The neuroprotective effects of the Allium cepa leaves extract is a virtue of its strong antioxidant activity.
\end{abstract}

Key words: Allium cepa, Neuropathy, $\mathrm{CCl}$, Oxidative stress, Sciatic nerve.

\section{INTRODUCTION}

Different animal models of neuropathy showed a strong correlation of sciatic nerve with neuropathic pain. Peripheral nerve injury models involve surgical procedures at sciatic nerve to induce neuropathy, whereas drug induced neuropathy and disease induced neuropathy are models developed due to oxidative stress, degeneration or neurotoxicity of peripheral nerves i.e sciatic nerve. ${ }^{1}$ Surgical models targeting nerve result in a chronic mechanical allodynia on injured paw. These models include chronic constriction injury, sciatic nerve cuffing, partial sciatic nerve ligation, spinal nerve ligation or common peroneal nerve ligation. ${ }^{2}$ While the disease induced neuropathy commonly include diabetic neuropathy model in research groups, ${ }^{1}$ chronic hyperglycemia induces oxidative stress through multiple pathways like redox imbalances, altered protein kinase $\mathrm{C}$ activity and mitochondrial overproduction of superoxide $^{3}$ and this plays a significant role in development of diabetic complications including diabetic neuropathy. ${ }^{4}$ While, sciatic nerve injury is associated with excessive production of reactive oxygen species, calcium ions entry and apoptosis; calcium overload through Transient Receptor Potential (TRP) cation channel and pain
Submission Date: 24-08-2019; Revision Date: 03-10-2019; Accepted Date: 23-11-2019

DOI: 10.5530/ijper.54.1.17 Correspondence: Dr. Hemant D Une, Y. B. Chavan College of Pharmacy, Dr. Rafiq Zakaria Campus, Dr. Rafiq Zakaria Marg, Rauza Bagh, Aurangabad-431001, Maharashtra, INDIA. Phone: +919823015556 E-mail: hemantdune@ rediffmail.com

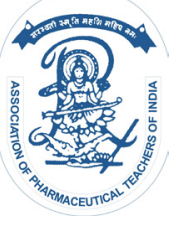

www.ijper.org 
intensity are associated with resulting oxidative stress following CCI. ${ }^{5}$

Being a reliable model of inducing diabetic neuropathy, Streptozotocin (STZ) in multiple sub-diabetogenic doses results in chronic hyperglycemia from partial damage to islets followed by an inflammatory process. In previous research work, it was found to develop diabetic neuropathy in rats. ${ }^{6}$ Chronic hyperglycemia and diabetic neuropathy leads to dysfunction and degeneration of the sciatic nerve. ${ }^{7}$

Allium cepa Lam. is a common and medicinally valuable plant. Leaves are reported to be aphrodisiac, anti-spasmodic, antihelmintic, alterative, carminative, digestive, diuretic, emollient, expectorant, mild laxative, stimulant and tonic along with presence of flavonoids, ${ }^{8}$ Vitamin A, thiamin and ascorbic acid. ${ }^{9}$ Outer layers of scales of its bulbs in different varieties were studied by researchers for their effects on chronic constriction injury induced neuropathic pain and resulted into significant antioxidant effect and improvement in neuropathic pain. ${ }^{10}$

In the present study flavonoid rich extract of $A$. cepa leaves was used to evaluate its efficiency for protecting sciatic nerve against neuropathy and oxidative stress by examining the extract effects on biomarkers of neuropathy in both surgical and diabetic neuropathy models followed by evaluation of oxidative stress after 5 weeks of diabetic neuropathy and 3 weeks of treatment and later animals were sacrificed to study histology of sciatic nerve.

\section{MATERIALS AND METHODS}

\section{Drugs and chemicals}

Streptozotocin (STZ, Spectrochem Pvt. Ltd.) was mixed in cold $0.01 \mathrm{M}$ citrate buffer, $\mathrm{pH}$ 4.5, prepared freshly before administration, ${ }^{6,11}$ and all other analytical grade chemicals were consumed in investigation.

\section{Plant material}

The leaves of $A$. cepa were collected from the local area of Aurangabad, Maharashtra, India. They were authenticated by Department of Botany, Dr. Babasaheb Ambedkar Marathwada University, Aurangabad, Maharashtra and a voucher specimen 0587 has been deposited in the herbarium of Dr. Babasaheb Ambedkar Marathwada University, Aurangabad (MS), India. Flavonoid rich extract was obtained from methanolic exract of $A$. cepa leaves followed by fractionation using ethyl acetate (ACEA- A. cepa: Ethyl acetate fraction) from the technique reported in earlier research work. ${ }^{6}$

\section{Experimental animals}

Sprague Dawley rats of either sex weighing between 180-250g were used. Prior to the experimental work, acclimatization of rats to the conditions of animal house for one week was carried out. Normal pellet diet and water ad libitum was followed for them. They were housed at standard conditions of temperature $\left(23 \pm 12^{\circ} \mathrm{C}\right)$, humidity $(45 \pm 5 \%)$ and $12 \mathrm{hr}$. light and dark cycle. The experimental protocol for animal experiments was approved by the Institutional Animal Ethics Committee (IAEC) of Y. B. Chavan College of Pharmacy, Aurangabad (Approval no. CPCSEA/IAEC/ P'col-52/115).

\section{Induction of Neuropathy Induction of experimental diabetic neuropathy}

Following overnight fasting experimental rats were injected with a multiple dose of $40 \mathrm{mg} / \mathrm{kg} /$ i.p. of STZ for 3 consecutive days. Soon within 5 min after mixing in citrate buffer $\mathrm{pH} 4.5$ the STZ solution was administered. The rats in group $\mathrm{NC}$ were injected with distilled water as a vehicle control. The animals were allowed to drink $5 \%$ glucose solution ad libitum overnight to overcome hypoglycemia. On inducing diabetes random blood glucose was estimated followed by regular analysis until attaining stable hyperglycemia. Rats may develop hyperglycemia and additional clinical diabetic indications within 3 days of STZ injection. After 1 week rats showing blood glucose above $250 \mathrm{mg} / \mathrm{dl}$ were prefered to study, 24 rats were selected for further studies. After 3 weeks of stable diabetes drug treatment was performed and pretreatment (pre $\mathrm{T} / \mathrm{T}$ ) studies of parameters of neuropathy were performed. On completion of dosing period rats were again tested for different parameters of Neuropathy. ${ }^{6,11,12}$

\section{Experimental Design}

Animals were distributed in various groups composed of six animals each. Group NC: Normal control, vehicle treated, Group DC: Diabetic control, vehicle treated, Group ACEA 25 : Diabetic, ACEA treated (25 mg/kg, p. o.), Group ACEA : : Diabetic, ACEA treated $\left(50 \mathrm{mg} / \mathrm{kg}\right.$, p. o.), Group ACEA $_{100}$ : Diabetic, ACEA treated (100 mg/kg, p. o.)

\section{Surgical Model of Neuropathy}

In order to obtain the surgical control group, partial sciatic nerve ligation technique was followed. Rats were allowed to fast overnight. Skin incision was made dorsal to pelvis followed by anaesthesia. The common sciatic nerve was first exposed by separating the muscles then loosely ligated with a chromic gut suture. Fascia, 
muscle and skin incision were closed using silk suture. After the completion of surgery and recovery of animals, the group was used for comparative study to assess tactile allodynia., ${ }^{2,13,14}$

\section{Experimental design}

Animals were allotted various groups with six animals in each group. Group NC: Normal control, vehicle treated, Group DC: Diabetic control, vehicle treated, Group ACEA $_{25}$ : Nerve ligation, ACEA treated (25 mg/kg, p. o.), Group ACEA 50 : Nerve ligation, ACEA treated (50 mg/kg, p. o.), Group ACEA ${ }_{100}$ : Nerve ligation, ACEA treated $(100 \mathrm{mg} / \mathrm{kg}$, p. o.)

\section{Blood Collection and blood glucose estimation}

Blood samples from the experimental groups were collected from tail vein. The samples so collected were analyzed for glucose estimation using FAD-GDH method by Contour TS glucometer. ${ }^{6,11}$

\section{Assessment of Behavioural Parameters Tactile Allodynia Von Frey Filament model}

Stimulus presentation and testing paradigms as described by S.R. Chaplan et al. was followed to obtain $50 \%$ threshold calculations and estimate tactie allodynia in rats. Animals were placed in acrylic chamber with wire mesh at bottom which gives full access to the paws. Animals were conceded to get acclimatized in the cage for about $15 \mathrm{~min}$. the paw was touched with vonfrey filaments following up-down method in order to calculate $50 \%$ withdrawal threshold. Vonfrey hairs were sujected to the paw perpendicularly with sufficient force to cause buckling response against paw and held for about 6-8 sec. Sharp withdrawal of paw was considered to be positive response. ${ }^{11,15,16}$

$50 \% \mathrm{~g}$ threshold was calculated by formula:

$$
\left(10^{[\mathrm{x} f \mathrm{k} \delta \mathrm{j}]}\right) / 10,000
$$

Where $x_{f}-$ Value (in log units) of final vonfrey hair used. k- Tabular value for the pattern of positive / negative responses (as provided in table) ${ }^{15}$

$\delta$ - Mean difference (in log units) between stimuli (here, $0.224)$

\section{Randall Selitto Analgesiometer}

Nociceptive withdrawal threshold was estimated on Randall selitto test apparatus on the paws of experimental animals. Hold animals with soft cotton cloth in order to immobilize it. Place gently the paw on the apparatus and allow the tip of device to apply on paw with application of increasing mechanical force and withdrawal latency to the pressure supported was noted down. ${ }^{11,17}$

\section{Assessment of Biochemical Parameters Glycosylated Haemoglobin}

Glycosylated Haemoglobin $\left(\mathrm{HbA}_{1 \mathrm{c}}\right)$ estimation was carried out by calorimetric method. Before sacrificing animals for biochemical and histopathological studies blood was collected using fine glass capillary puncturing of retro-orbital plexus in epindorff tubes. Serum and packed blood cells were separated by centrifugation for collecting RBCs. The packed cells were washed 3-4 times in normal saline. After final wash distilled water and $\mathrm{CCl}_{4} 0.5 \mathrm{ml}$ each were added and mixed vigorously followed by $20 \mathrm{~min}$ centrifugation at $3000 \mathrm{rpm}$. The supernatant haemolysate were separated and its $\mathrm{Hb}$ concentration was adjusted to $10 \mathrm{gms} \%$ with distill water. To $2 \mathrm{ml}$ of haemolysate, $1 \mathrm{ml}$ of $0.3 \mathrm{~N}$ oxalic acid was added and kept in boiling water bath for 60 min after covering the test tube using cotton plug. After cooling $1 \mathrm{ml}$ of $40 \%$ TCA was added, shaken vigorously and centrifuged at $3000 \mathrm{rpm}$. To $2 \mathrm{ml}$ of this supernatant $0.5 \mathrm{ml}$ of $0.05 \mathrm{M}$ thiobarbituric acid was added and kept at $37^{\circ} \mathrm{C}$ for $40 \mathrm{~min}$. The resultant yellow color was read on colorimeter at $443 \mathrm{~nm}$, reading was taken as blank $2 \mathrm{ml}$ distilled water and $0.5 \mathrm{ml}$ of thiobarbituric acid $\left(\mathrm{HbA}_{1 \mathrm{c}}\right.$ was calculated on assumption that $1 \% \mathrm{HbA}_{1 \mathrm{c}}$ corresponds to an absorbance of 0.029 at $443 \mathrm{~nm}) ., 18$

\section{Estimation of markers of oxidative stress}

At end of the treatment and behavioral assessments, animals were sacrificed under deep anesthesia preceded by overnight fasting. By using Remi homogenizer, sciatic nerves on rapid removal were homogenized in a cold $50 \mathrm{mM}$ phosphate buffered saline ( $\mathrm{pH}$ 7.4). The $10 \% \mathrm{w} / \mathrm{v}$ tissue homogenate was centrifuged at 1000 $\mathrm{rpm}$ for $20 \mathrm{~min}$ at $4^{\circ} \mathrm{C}$ for estimation of catalase activity while for SOD and GSH homogenate was centrifuged at $12000 \mathrm{rpm}$ as long as $60 \mathrm{~min}$ at $4^{\circ} \mathrm{C}$.Catalase activity was estimated applying $\mathrm{H}_{2} \mathrm{O}_{2}$ just as substrate. In brief, $\mathrm{H}_{2} \mathrm{O}_{2}$ decomposition by catalse was monitored following the decrease in absorbance at $240 \mathrm{~nm} .{ }^{19} \mathrm{SOD}$ activity in sciatic nerve was estimated by its ability to inhibit auto-oxidation of Pyrogallol at $420 \mathrm{~nm} .{ }^{20}$ Concentration of GSH was estimated spectrophotometrically using DTNB reagent at $412 \mathrm{~nm} .^{21,22}$

\section{Histopathological evaluation}

Animals were sacrificed and the sciatic nerve was carefully removed from each animal. The nerve tissue was washed with saline and fixed in 10\% formalin for histopathological study. Slides were prepared by embedding 
the nerve in paraffin and staining with hematoxylin and eosin. ${ }^{23}$ The histology of sciatic nerves was studied for axonal degeneration, dilatation, edema and vacuolization using a bright field microscope and images were taken for reference. ${ }^{24}$

\section{Statistical Analysis}

All the data expressed by Mean $\pm \operatorname{SEM}(n=6)$, twoway ANOVA, followed by Benferroni test using Prism Graph Pad version 5 with $p$ value $<0.001,<0.05$ and $<0.01$ statistical significance.

\section{RESULTS}

In the previous research work it was reported that ACEA fraction is flavonoids rich having presence of quercetin with a potential ant diabetic activity and protective in thermal hyperalgesia, cold allodynia and motor inco-ordination behavioral biomarkers of diabetic neuropathy.

\section{Effects of ACEA on mechanical allodynia (Vonfrey filaments) in neuropathic pain.}

Nociceptive withdrawal threshold expressed in terms of $50 \%$ threshold of diabetic control and surgical control was significantly $(p<0.001)$ decreased comparable with that of normal control. The ACEA treated groups shows dose dependant significant $(p<0.05,0.001)$ increase in paw withdrawal threshold comparable with that of diabetic and surgical control (Table 1).

\section{Effects of ACEA on Mechanical or Static Hyperalgesia (Randall Selitto test) in neuropathic pain.}

Nociceptive withdrawal threshold expressed in terms of supported pressure resulting from application of randall-selitto probe of diabetic and surgical control has found to decrease significantly $(p<0.001)$ comparable with that of normal control while ACEA has shown dose dependant increase in supported pressure comparable with that of diabetic and surgical control significantly $(\phi<0.001)$, (Table 1$)$.

\section{Effects of ACEA on Glycosylated Haemoglobin level in neuropathic pain.}

The $\mathrm{HbA}_{1 \mathrm{c}}(\%)$ value of diabetic control has found to increase significantly $(p<0.001)$ comparable with that of normal control while ACEA has shown dose dependant decrease in value comparable with that of diabetic control significantly $(p<0.05, p<0.001)$, (Figure 1).

\section{Effects of ACEA on oxidative stress markers in neuropathic pain.}

The biomarkers of oxidative stress were assessed, ACEA dose dependently increased the Catalase, SOD and GSH levels significantly $(p<0.01, p<0.001)$ in sciatic nerve comparable with that of diabetic and surgical control group (Figure 2) thus, resulted in normalization of STZ as well as CCI induced biochemical abnormalities in a significant manner.

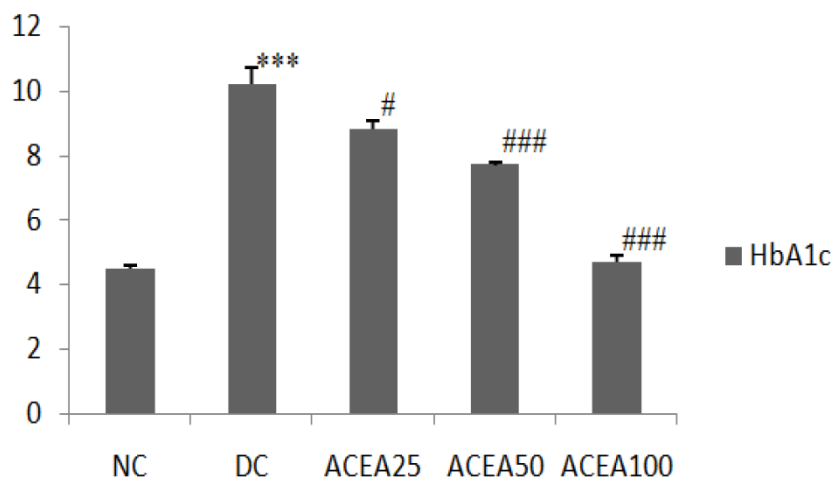

Figure 1: Effect of ACEA on $\mathrm{HbA}_{1 \mathrm{c}}$ value.

Data is represented in the form of Mean $\pm \operatorname{SEM}(n=6)$, Two way ANOVA followed by Benferroni test using Prism Graph Pad version 5 with $* p<0.05$ and $* * * p<0.001$ significance for groups are compared with normal group while $\# p<0.05$ and $\# \# \# p<0.001$ for groups compared with diseased group. (NC-Normal Control, DCDiabetes Control, $\mathrm{ACEA}_{25}$ - Extract treated, $25 \mathrm{mg} / \mathrm{kg}$ p.o., $\mathrm{ACEA}_{50}$ - Extract treated, $50 \mathrm{mg} / \mathrm{kg}$ p.o. and $\mathrm{ACEA}_{100}$ - Extract treated, $100 \mathrm{mg} / \mathrm{kg}$ p.o.)

\begin{tabular}{|c|c|c|c|c|c|}
\hline \multirow{2}{*}{ Tactile Allodynia } & \multicolumn{5}{|c|}{ Surgical induced neuropathy } \\
\hline & NC & SC & ACEA25 & ACEA50 & ACEA100 \\
\hline Vonfrey Filaments ( $50 \%$ threshold (gm)) & $8.81 \pm 0.7$ & $1.72 \pm 0.1^{* * *}$ & $3.41 \pm 0.2^{\# \#}$ & $4.35 \pm 0.1 \ldots \ldots$ & $5.7 \pm 0.2^{\# \#}$ \\
\hline \multirow[t]{3}{*}{ Randall-Sellito apparatus (Supported Pressure (gm)) } & $318.06 \pm 18.9$ & $82.54 \pm 3.2^{* * *}$ & $123.13 \pm 4$ & $140.60 \pm 1.5$ & $234.80 \pm 28.2^{\# \#}$ \\
\hline & \multicolumn{5}{|c|}{ STZ induced neuropathy } \\
\hline & $\mathrm{NC}$ & $\mathrm{DC}$ & ACEA25 & ACEA50 & ACEA100 \\
\hline Vonfrey Filaments ( $50 \%$ threshold $(\mathrm{gm})$ ) & $8.81 \pm 0.7$ & $1.37 \pm 0.09^{* * *+}$ & $3.36 \pm 0.2^{\#}$ & $4.20 \pm 0.1^{\# \# \#}$ & $5.61 \pm 0.2^{\# \#}$ \\
\hline Randall-Sellito apparatus (Supported Pressure (gm)) & $318.06 \pm 18.9$ & $81.16 \pm 3.4^{* *+x}$ & $122.84 \pm 4.1$ & $140.23 \pm 1.3$ & $234.49 \pm 28.6^{\# \#}$ \\
\hline
\end{tabular}

Data is represented in the form of Mean $\pm \operatorname{SEM}(n=6)$, Two way ANOVA followed by Benferroni test using Prism Graph Pad version 5 with $* p<0.05$ and $* * * p<0.001$ significance for groups are compared with normal group while $\# p<0.05$ and \#\#\#p<0.001for groups compared with diseased group. 


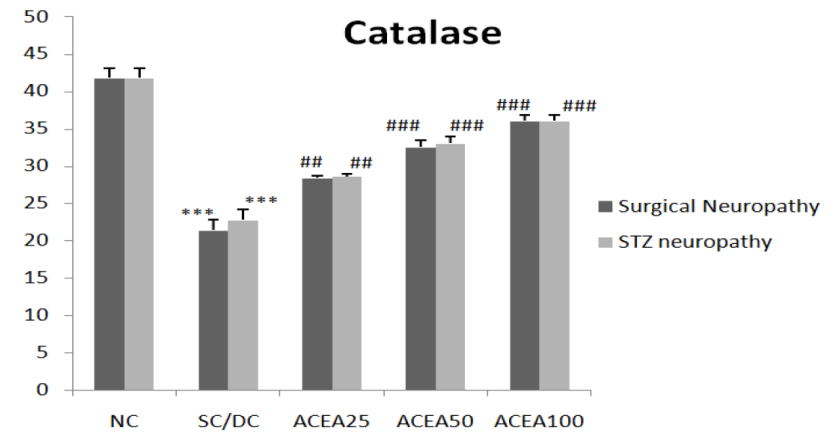

A

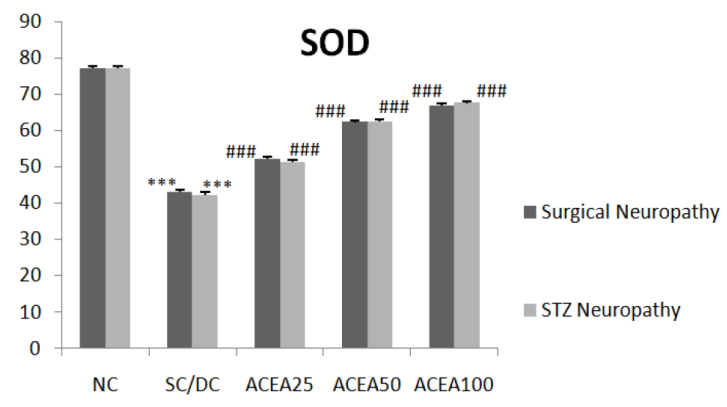

B

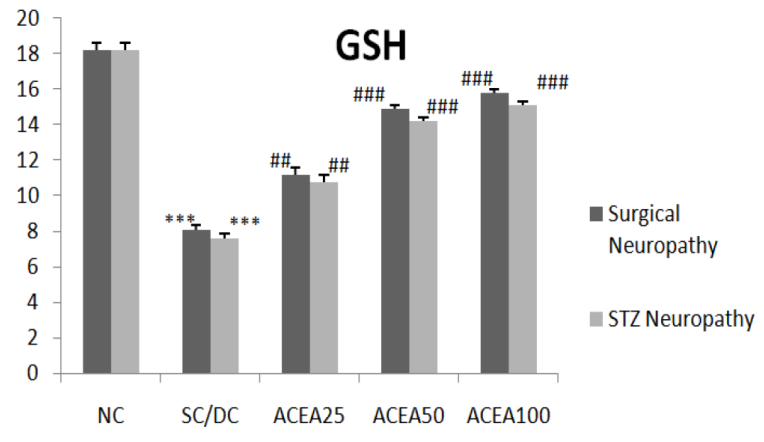

C

Figure 2: Effect of ACEA on A-Catalase, B-SOD and C-GSH markers of oxidative stress on Sciatic nerve.

Data is represented in the form of Mean \pm SEM $(n=6)$, Two way ANOVA followed by Benferroni test using Prism Graph Pad version 5 with** $p<0.01$ and $* * * p<0.001$ significance for groups are compared with normal group while $\# p<0.05$ and $\# \# \# p<0.001$ for groups compared with diseased group. (NC-Normal Control, SC/ DC-Surgical Control/Diabetes Control, ACEA $_{25}$ - Extract treated, $25 \mathrm{mg} / \mathrm{kg}$ p.o.,

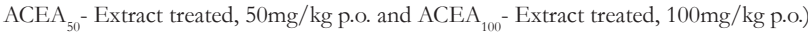

\section{Effects of ACEA on Histopathological changes in sciatic nerve.}

All of the histopathological parameters of sciatic nerves are shown in Figure 3. In diabetic control and surgical control groups, it was determined severe axonal degenerations; mild dilatations and edema were observed in nerve epineuriums. While ACEA treated group depicted repaired sciatic nerve with no axonal degenerations, dilatation, edema and vacuolization.

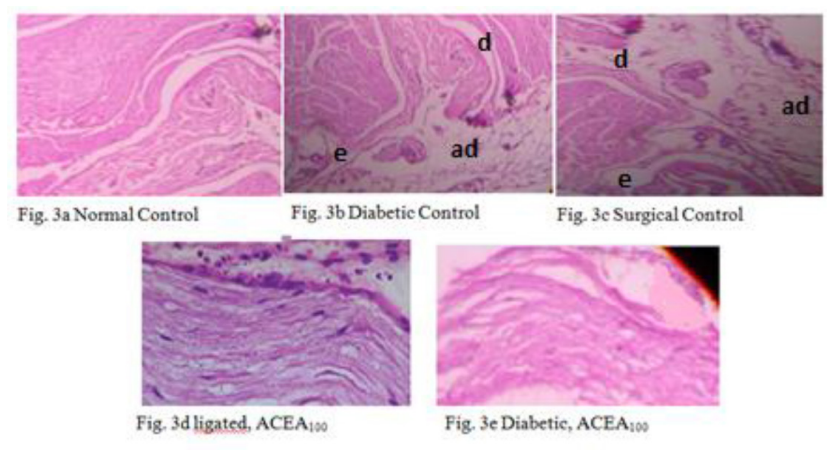

Figure 3: Histopathology study of Sciatic nerve with $H$ and $E$ stain, where ad: axonal degeneration, d: dilatation and e: edema. (ACEA ${ }_{100}$ - Extract treated, $100 \mathrm{mg} / \mathrm{kg}$ p.o.)

\section{DISCUSSION}

Different animal models for neuropathic pain circles around sciatic nerve dysfunction, ${ }^{1}$ from which the surgical model and diabetes induced neuropathy were successfully followed in the present work. The surgical nerve injury model mimics symptoms of chronic nerve compression in humans. ${ }^{14}$ Previous research work has proved STZ induced diabetic neuropathy model to be reliable and widely accepted model and has also proven ACEA to be a flavonoid rich extract ${ }^{6,11}$ also it was found that free radical generation and oxidative stress has long been involved in neuropathic pain pathogenesis. ${ }^{5,14}$ On the basis of previous reports of signs of STZ induced diabetic model ${ }^{14}$ after one week from induction of diabetes, animals were tested for stable hyperglycemia and hyperalgesia and were used for further studies. In tactile allodynia quantitative assessment is estimated by Vonfrey filaments and Randall selitto test, $50 \%$ threshold for nociceptive withdrawal latency provides quantitative assessment of tactile allodynia expressed by Vonfrey filaments. Randall selitto is another adequate and sensitive method to quantify tactile allodynia in neuropathy, threshold for withdrawal latency is measured in the form of supported pressure in randall selitto test. 2,11,15 Thus, in the present study tactile allodynia has been successfully quantified and proved to be useful and versatile techniques for assessment of appearance of neuropathic pain.

Glycosylated Haemoglobin $\left(\mathrm{HbA}_{1 \mathrm{c}}\right)$ is a result of haemoglobin molecule undergoing post translational changes, their levels correlate with glycemic levels over the previous six to ten weeks. Under physiological condition, a reaction between glucose and $\mathrm{N}$ terminal valine of Beta chain of $\mathrm{Hb}$ molecules proceeds to glycosylation of haemoglobin. ${ }^{18}$ In the existing study this parameter was used to support STZ induced 
neuropathy and it was found ACEA has attenuating action over it too (Figure 1).

It has been reported that sciatic nerve ligation induces imbalance in reactive oxygen species and antioxidant enzymes also oxidative stress has been noted to induced by hyperglycemia. ${ }^{3,25}$ In our study, ACEA has shown restoration of biochemical parameters such as catalase, SOD and GSH level in nerve tissues.

It is well established by previous reports that chronic hyperglycema induces oxidative stress and proves to be neurotoxic that leads to neuronal apoptosis. ${ }^{26}$ These neuronal damages can be studied with the help of morphological study of sciatic nerve. Histopathology study of sciatic nerve highlighting axonal degeneration, dilatation, edema and vacuolization acts as degree of nerve damage ${ }^{24}$ due to surgical and diabetes induced neuropathy were obtained in results. Present work determines histopathology of sciatic nerve of diabetic control and surgical control groups showed significant derangement of nerve cells with signs of axonal degeneration, dilatation and edema while that of ACEA treated group shows signs of repair of nerve cells from structural alteration as there are no signs of degeneration seen as compared to surgical and diabetic control groups (Figure 3). Improved nerve structures are considered as a marker for protective action of extract on damaged nerve. ${ }^{23}$

\section{CONCLUSION}

Surgical as well as STZ induced neuropathy model were found successful in inducing neuropathy and oxidative stress in present work. ACEA has depicted protective action in biomarkers of tactile allodynia, oxidative stress; glycosylated haemoglobin and confirmation of protective effects were obtained through histopathological study of sciatic nerve. ACEA has significantly proven to have ameliorative potential over STZ and CCI induced neuropathic pain addressing it as a better approach to drug development in treatment of neuropathy.

\section{ACKNOWLEDGEMENT}

The authors are thankful to the Principal, Dr. Zahid Zaheer, Y. B. Chavan College of Pharmacy, Aurangabad for providing all the facilities required periodically for the study.

\section{CONFLICT OF INTEREST}

The authors declare no conflict of interest.

\section{ABBREVIATIONS}

ACEA: Ethyl acetate fraction from methanolic extract of leaves of $A$. cepa Lam; FAD-GDH: Flavin Adenine Dinucleotide- Glucose Dehydrogenase.

\section{REFERENCES}

1. Amteshwar SJ, Vivek J, Nirmal S. Animal models of neuropathic pain. Fundament and Clin Pharmacol. 2011;25(1):1-28.

2. Ipek $Y$, Salim M, Florent B, Elisabeth $W$, Mélanie K, Eric S, et al. The Sciatic Nerve Cuffing Model of Neuropathic Pain in Mice. J of Visual Exp. 2014;89:1-7.

3. Netaji T, Dipak GP, Rahul SS, Rajkumari SS. Effect of rutin on early diabetic neuropathy in experimental animals. J Nat Prod Plant Resour. 2014;4(4):1-9.

4. Ferdinando G, Michael B. Oxidative stress and diabetic complications. Circ res. 2010;107(9):1058-70.

5. Uslusoy F, Naziroglu M, Cig B. Inhibition of the TRPM2 and TRPV1 Channels through Hypericum perforatum in Sciatic Nerve Injury-induced Rats Demonstrates their Key Role in Apoptosis and Mitochondrial Oxidative Stress of Sciatic Nerve and Dorsal Root Ganglion. Front Physiol. 2017;8:1-16.

6. Khan D, Mohammed M, Hemant DU. Quantification of Quercetin obtained from Allium cepa Lam. leaves and its effects on Streptozotocin induced diabetic neuropathy. Pharmacog Res. 2017;9(3):287-93.

7. Maher MA. Ameliorative potential of rutin on streptozotocin induced neuropathic pain in rat. Afric J of Pharm and Pharmacol. 2013;7(41):2743-54.

8. Ashwini M, Satishkumar R. Onion (Allium cepa) - Ethnomedicinal and therapeutic properties: Handbook of Medicinal Plants and their Bioactive Compounds. Research Signpost. 2014;27-34.

9. Sampath KKP, Debjit B, Chiranjib B, Pankaj T. Allium cepa: A traditional medicinal herb and its health benefits. J Chem Pharm Res. 2010;2(1):283-91.

10. Amit K, Kundan SB, Amteshwar SJ, Richa S. Comparative evaluation of neuropretective effect of three varieties of Allium cepa in chronic constriction injury induced neuropathic pain. Thai J of Pharmaceu Sci. 2016;40(1):9-20.

11. Dureshahwar K, Mubashir M, Upaganlawar A, Sangshetti JN, Upasani CD, Une HD. Quantitative assessment of tactile allodynia and protective effects of flavonoids of Ficus carica Lam. leaves in diabetic neuropathy. Phcog Mag. 2019;15(62 Suppl 1):128-34.

12. Kiran HB, Aman BU. Neuroprotective efects of Lagerstroemia speciosa L. extract (Banaba leaf extract) in streptozotocin induced painful diabetic neuropathy in laboratory rats. Pharmacol. 2016;7(1):9-15.

13. Kiran HB, Aman BU, Chandrashekhar DU. Alcoholic extract of banaba leaf prevents chronic constriction injury induced neuropathic pain in experimental animals. Annals of Med and Biomed Sci. 2016;2(1):52-6.

14. Shaikh AS, Somani RS. Animal models and biomarkers of neuropathy in diabetic rodents. Ind J of Pharmacol. 2010;42(3):129-34.

15. Chaplan SR, Bach FW, Pogrel JW, Chung JM, Yaksh TL. Quantitative assessment of tactile allodynia in the rat paw. $\mathrm{J}$ of Neurosci Methods. 1994;53(1):55-63.

16. Sanklecha D, Upaganlawar A, Upasani C. Neuroprotective Effects of Protocatechuic Acid In Diabetes Induced Neuropathic Pain. Am J of Biochem and Mol Bio. 2017;7(3):111-7.

17. Eva S, Elena RC, Xavier N. Randall-Selitto Test: A new approach for the detection of Neuropathic Pain after Spinal Cord Injury. J of Neurot. 2012;29(5):898-904

18. Ishrat K, Jaweed SA, Bardapurkar JS, Patil VP. Study of magnesium, glycosylated hemoglobin and lipid Profile in diabetic retinopathy. Ind J of Clin Biochem. 2004;19(2):124-7.

19. Clairborne A. Catalase activity: Handbook of methods for oxygen radical research. Boca Raton: CRC Press. 1985;283-4.

20. Marklund SL. Pyrogallol autooxidation: Handbook of methods for oxygen radical research. Boca Raton: CRC Press. 1985;243-47.

21. Sedlak J, Lindsay RH. Estimation of total, protien-bound and nonprotien sulfhydryl groups in tissue with Ellman's reagent. Analyt Biochem. 1968;25(1):192-205

22. Ellman GL. Tissue sulfhydryl groups. Arch Biochem Biophys. 1959;82(1):70-7.

23. Parkar N, Addepalli V. Effect of Nobiletin on diabetic neuropathy in experimental rats. Austin J of Pharmacol Ther. 2014;2(5):1-5. 
mir A, Kalkan Y, Bostan H. Histopathological effects of intramuscula metamizole sodium on sciatic nerve. Iran J Basic Med Sci. 2016;19(8):829-36. sciatic nerve ligation induced-neuropathic pain. Int $\mathrm{J}$ of Pharmaceut Sci and Res. 2012;3(2):509-18.
26.

Stevens MJ, Obrosova I, Cao X, Huysen CV, Greene DA. Effects of dl- $\alpha$ lipoic acid on peripheral nerve conduction, blood flow, energy metabolism and oxidative stress in experimental diabetic neuropathy. Diabetes. 2000;49(6):1006-15.

\section{PICTORIAL ABSTRACT}

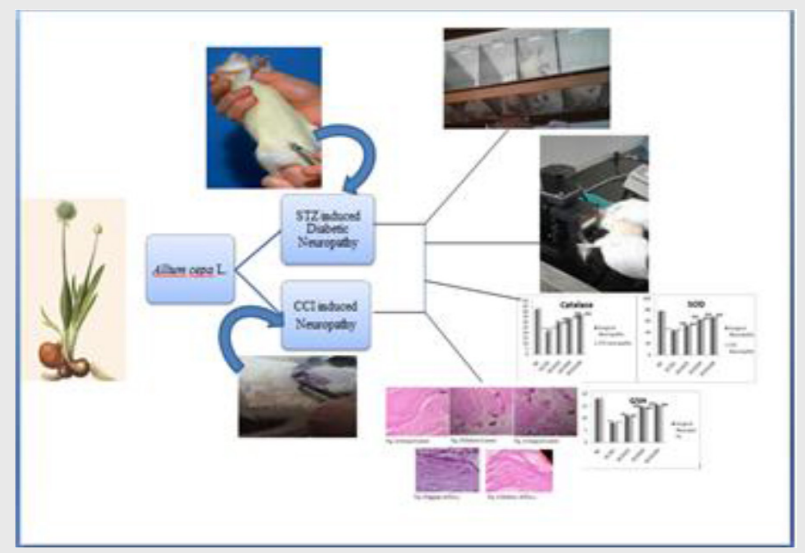

\section{SUMMARY}

The current research work shows surgical as well as STZ induced neuropathy model to be successful in inducing neuropathy and oxidative stress. Leaves of Allium cepa L. provides protective action in quantitative assessment of tactile allodynia, oxidative stress; glycosylated haemoglobin and histopathological study of the sciatic nerve. This plant sample has proven ameliorative potential in STZ and CCI induced neuropathic pain.

\section{About Authors}

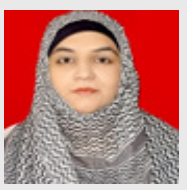

Dureshahwar Khan, working as Assistant Professor, Department of Pharmacology, Y. B. Chavan College of Pharmacy, Rouza Bagh, Aurangabad, Maharashtra, India. She has one patent application published, 17 citations and $3 \mathrm{~h}$-index to her credit. She has a core competency in the extraction of herbal drugs, molecular pharmacology and Neuropharmacology.

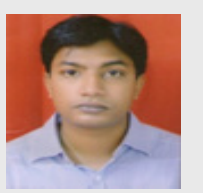

Mohammed Mubashir, Research Scholar, Department of Pharmacology, Y. B. Chavan College of Pharmacy, Rouza Bagh, Aurangabad, Maharashtra, India. He has one patent application published, 17 citations and $3 \mathrm{~h}$-index to his credit. His research interest includes diabetic neuropathy, herbal extractions and anti cancer activity.

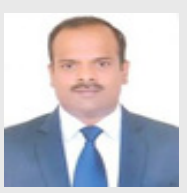

Aman B. Upaganlawar, working as Associate Professor, SNJBs SSDJ College of Pharmacy, Chandwad, Nashik, Maharashtra, India. He has 1139 citations, 18 h-index and 27 i10-index to his credit. His area of research includes cardiovascular and biochemical pharmacology, screening of bioactive materials for various activities and diabetes and its complications.

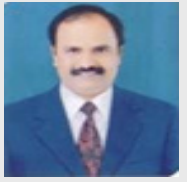

Chandrashekhar D. Upasani, working as Professor of Pharmacology and Principal, SNJB's Shriman Sureshdada Jain College of Pharmacy, Chandwad, Nashik, Maharashtra, India. He has 827 citations, $14 \mathrm{~h}$-index and $19 \mathrm{i} 10$-index to his credit. His research interests include cardiovascular system, autonomic nervous system, role of heavy metals and metalloids in pathophysiology of various diseases, role of oxidative stress in behavioral, systemic and inflammatory responses of animals, the cellular and molecular basis of oxidative stress and endocrine pharmacology.

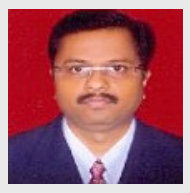

Hemant Devidas Une, Working as Associate Professor, Head of Department of Pharmacology, Y. B. Chavan College of Pharmacy, Rouza Bagh, Aurangabad, Maharashtra, India. He has one patent application published, 261 citations, 9 h-index and 9 i10-index to his credit. He has a core competency in preclinical evaluations for CNS acting agents, anti-diabetics and immunomodulators, herbal extraction and isolation. He has many research grants to his credit from government agencies.

Cite this article: Khan D, Mohammed M, Upaganlawar A, Upasani CD, Une HD. Ameliorative Potential of Allium cepa Lam. Leaves on Diabetes Induced and Chronic Constriction Injury Induced Neuropathic Pain in Experimental Rats. Indian J of Pharmaceutical Education and Research. 2020;54(1):143-9. 